\title{
Re-emerging Infectious Disease (RED) Alert tool
}

\author{
Maneesha Chitanvis ${ }^{\star 1}$, Ashlynn Daughton ${ }^{1,2}$, Forest M. Altherr ${ }^{1}$, Geoffrey Fairchild ${ }^{1}$, \\ William Rosenberger ${ }^{1}$, Emily Alipio Lyon ${ }^{1}$, Attelia Hollander ${ }^{1}$, Nileena Velappan ${ }^{1}$, \\ Derek Aberle ${ }^{1}$, Nidhi Parikh ${ }^{1}$ and Alina Deshpande ${ }^{1}$
}

${ }^{1}$ Biosecurity and Public Health, Los Alamos National Laboratory, Los Alamos, NM, USA; 2 University of Colorado, Boulder, Boulder, CO, USA

\section{Objective}

Although relying on verbal definitions of "re-emergence", descriptions that classify a "re-emergence" event as any significant recurrence of a disease that had previously been under public health control, and subjective interpretations of these events is currently the conventional practice, this has the potential to hinder effective public health responses. Defining re-emergence in this manner offers limited ability for ad hoc analysis of prevention and control measures and facilitates non-reproducible assessments of public health events of potentially high consequence. Re-emerging infectious disease alert (RED Alert) is a decision-support tool designed to address this issue by enhancing situational awareness by providing spatiotemporal context through disease incidence pattern analysis following an event that may represent a local (country-level) re-emergence. The tool's analytics also provide users with the associated causes (socioeconomic indicators) related to the event, and guide hypothesisgeneration regarding the global scenario.

\section{Introduction}

Definitions of "re-emerging infectious diseases" typically encompass any disease occurrence that was a historic public health threat, declined dramatically, and has since presented itself again as a significant health problem. Examples include antimicrobial resistance leading to resurgence of tuberculosis, or measles re-appearing in previously protected communities. While the language of this verbal definition of "re-emergence" is sensitive enough to capture most epidemiologically relevant resurgences, its qualitative nature obfuscates the ability to quantitatively classify disease re-emergence events as such.

\section{Methods}

Our tool automatically computes historic disease incidence and performs trend analyses to help elucidate events which a user may considered a true re-emergence in a subset of pertinent infectious diseases (measles, cholera, yellow fever, and dengue). The tool outputs data visualizations that illustrate incidence trends in diverse and informative ways. Additionally, we categorize location and incidence-specific indicators for re-emergence to provide users with associated indicators as well as justifications and documentation to guide users' next steps. Additionally, the tool also houses interactive maps to facilitate global hypothesis-generation.

\section{Results}

These outputs provide historic trend pattern analyses as well as contextualization of the user's situation with similar locations. The tool also broadens users' understanding of the given situation by providing related indicators of the likely re-emergence, as well as the ability to investigate re-emergence factors of global relevance through spatial analysis and data visualization.

\section{Conclusions}

The inability to categorically name a re-emergence event as such is due to lack of standardization and/or availability of reproducible, data-based evidence, and hinders timely and effective public health response and planning. While the tool will not explicitly call out a user scenario as categorically re-emergent or not, by providing users with context in both time and space, RED Alert aims to empower users with data and analytics in order to substantially enhance their contextual awareness; thus, better enabling them to formulate plans of action regarding re-emerging infectious disease threats at both the country and global level.

\section{Keywords}

Infectious Disease; Re-emergence; Pattern analysis; Contextual Awareness

\section{Acknowledgments}

This work was supported by the Defense Threat Reduction Agency's Joint Science and Technology Office for Chemical and Biological Defense under project numbers CB10007. Los Alamos National Laboratory is operated by Los Alamos National Security, LLC for the Department of Energy under contract DE-AC52-06NA25396. The authors also wish to thank Bryan Lewis at the Biocomplexity Institute of Virginia Tech and Ramesh Krishnamurthy at the World Health Organization. LA-UR-1721884 .

\section{*Maneesha Chitanvis}

E-mail: mchitanvis@lanl.gov 